\title{
Direct Write Protein Patterns for Multiplexed Cytokine Detection From Live Cells Using Electron Beam Lithography
}

Uland Y. Lau, ${ }^{a}$ Sina S. Saxer,,$^{b, \dagger}$ Juneyoung Lee, ${ }^{b}$ Erhan Bat,${ }^{b,}$ Heather D. Maynard ${ }^{* a, b}$

${ }^{a}$ Department of Bioengineering, University of California, Los Angeles, 410 Westwood Plaza, Los Angeles, California 90095, United States

${ }^{b}$ Department of Chemistry and Biochemistry and the California NanoSystems Institute, University of California, Los Angeles, 607 Charles E. Young Drive East, Los Angeles, California 90095, United States

${ }^{\dagger}$ Present Address: Department of Chemistry and Biochemistry, University of Applied Sciences and Arts, Northwestern Switzerland, Muttenz 4132, Switzerland

Present Address: Department of Chemical Engineering, Middle East Technical University, Ankara 06800, Turkey

\section{Supporting Information}


a

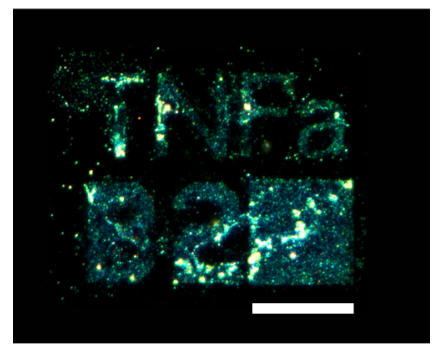

b

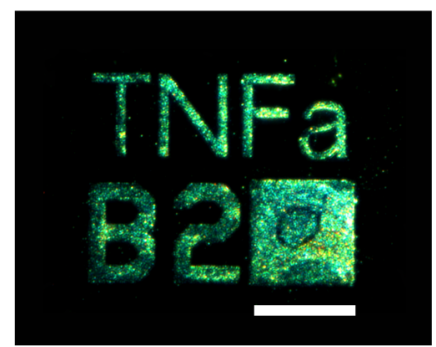

C

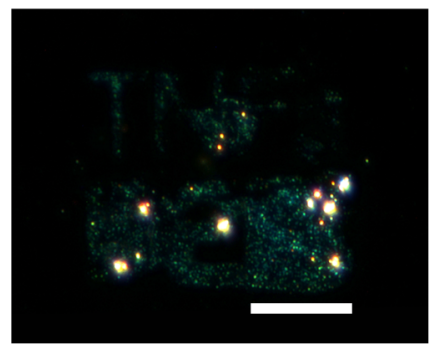

Figure S1. Effect of ascorbic acid on anti-TNF $\alpha$ patterning and immunoassay signals. AntiTNF $\alpha$ patterned with area dose of $25 \mu \mathrm{C} / \mathrm{cm}^{2}$ with $0.5 \% \mathrm{wt} / \mathrm{vol}$ PolyProtek and ascorbic acid concentration of a) $0 \mathrm{mM}$, b) $1 \mathrm{mM}$, and c) $2 \mathrm{mM}$. Scale bars $=35 \mu \mathrm{m}$.
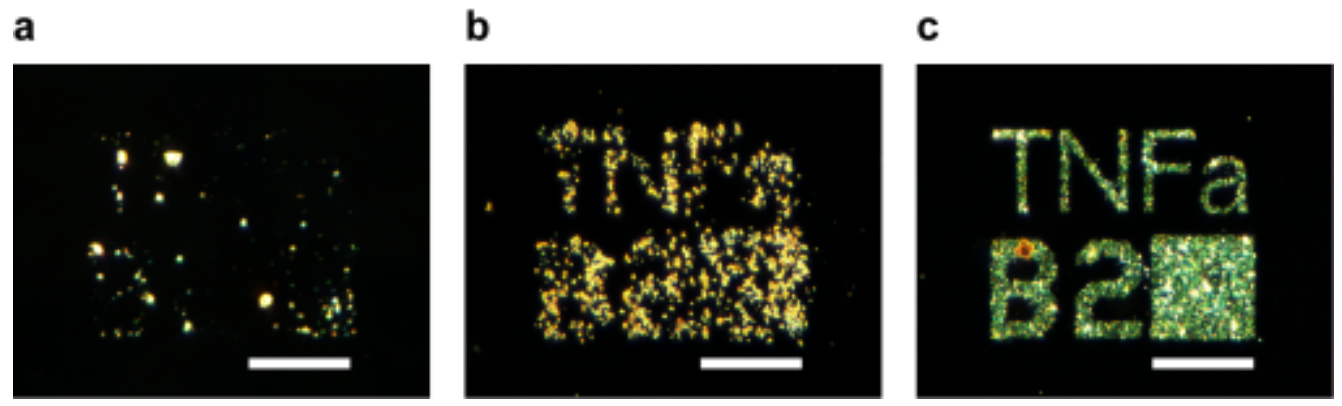

Figure S2. The effect of anti-TNFa spin-coating concentration on immunoassay signals. Concentrations of anti-TNF $\alpha$ used for spin-coating at a) $1 \mu \mathrm{M}$, b) $2 \mu \mathrm{M}$, and c) $5 \mu \mathrm{M}$. Scale bar $=35 \mu \mathrm{m}$. 
a
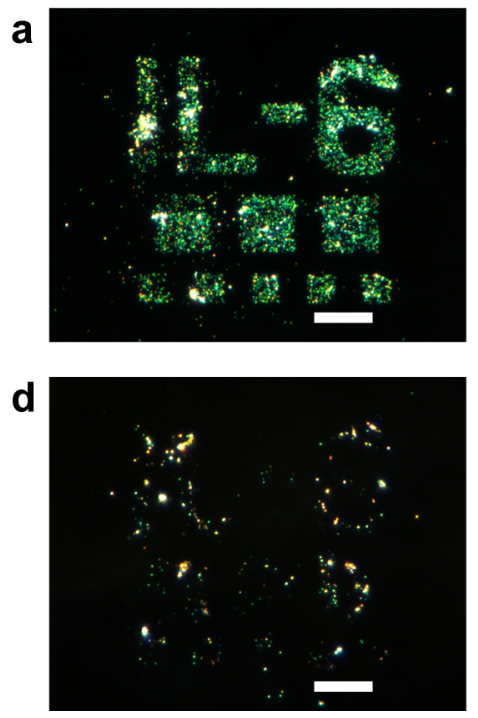

b

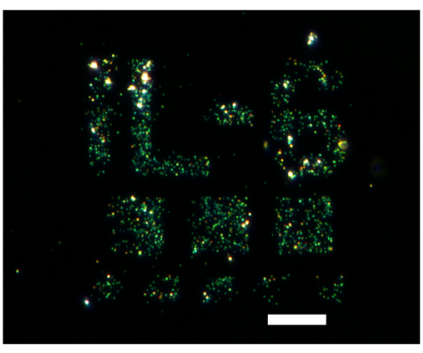

e

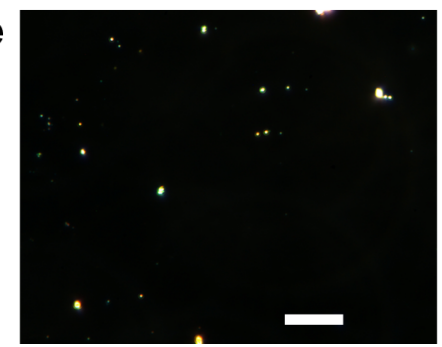

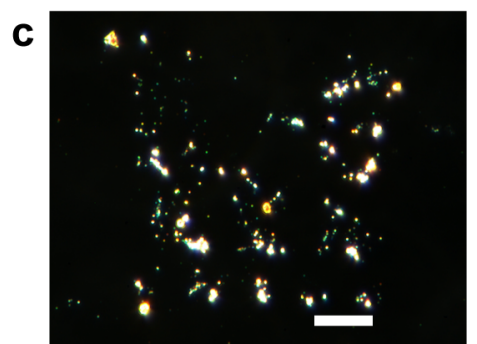

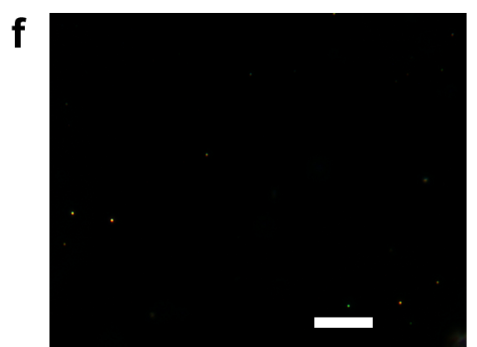

Figure S3. Detection sensitivity of anti-IL-6 patterns to varying concentrations of IL-6 in media: a) $200 \mathrm{ng} / \mathrm{mL}$, b) $50 \mathrm{ng} / \mathrm{mL}$, c) $500 \mathrm{pg} / \mathrm{mL}$, d) $50 \mathrm{pg} / \mathrm{mL}$, e) $5 \mathrm{pg} / \mathrm{mL}$, and f) 0 . Scale bars $=20$ $\mu \mathrm{m}$.

a

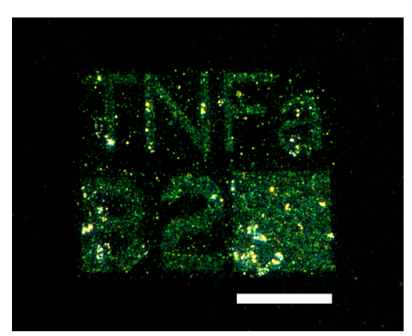

b

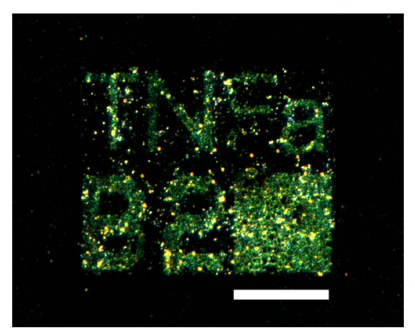

C

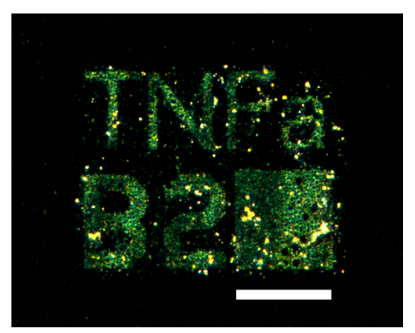

d

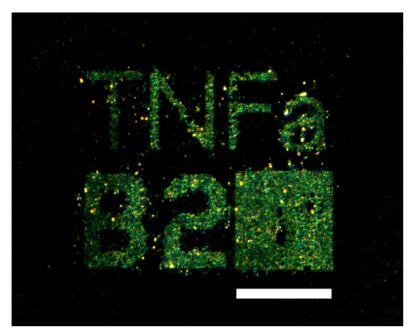

Figure S4. Repeated processing and exposure cycles of anti-TNF $\alpha$ patterned on the same substrate. The same substrate was subjected to four cycles of spin-coating with anti-TNF $\alpha$ antibody, e-beam patterning, and rinsing, and then developing with the immunoassay. Dark field micrographs show the pattern from the a) first cycle, b) second cycle, c) third cycle, and d) fourth cycle. The signal-to-noise of the square in the first cycle was 20.9 and in the fourth cycle 23.4 calculated as (signal from the box - signal of the background) / (stdev of the background). Scale bars $=35 \mu \mathrm{m}$. 